\title{
7 The trouble with definitions and the aims of conceptual analysis
}

Up to this point, a considerable part of this work has been dedicated to scrutability - the thesis that if we are given sufficient, nontrivial information about the character of a world, we are able to determine the extensions of our expressions. The following version of the thesis was mainly defended in the first part of chapter 6:

(CJ) If a subject possesses a concept and has unimpaired rational processes, then sufficient empirical information about the actual world puts a subject in a position to identify the concept's extension.

Before that, in 4.1, I argued for the conditional claim that if one endorses $(\mathrm{CJ})$, then one should also accept $(\mathrm{CJ}++)$ :

(CJ++) If a subject possesses a concept and has unimpaired rational processes, then sufficient information about any given scenario puts a subject in a position to identify the concept's extension with respect to that scenario a priori.

Since these theses involve a number of idealizations, I also discussed, in the second part of chapter 6, to what extent we are able to make such judgments in practice. My verdict was overall rather optimistic, since it turned out that there are at least no good reasons to be skeptical about our ability to evaluate hypothetical scenarios.

One obvious reason for dedicating so much space to a defense of scrutability is that if one manages to establish $(\mathrm{CJ}++)$, one has thereby shown that linguistic expressions are associated with an a priori accessible semantic value. The relevance of this result for the prospects of conceptual analysis should be obvious. A second, more specific reason for making scrutability a central theme is that it is ideally suited to provide the foundations for the way conceptual analysis is usually pursued, namely via thought experiments, i.e. the construction and evaluation of hypothetical scenarios. 
These observations leave open, however, what kinds of philosophical insights one may hope to gain on the basis of our grasp of primary intensions and our associated ability to evaluate hypothetical cases. This chapter will therefore be dedicated to what conceptual analysis might aim at.

A common source of skepticism towards conceptual analysis is the absence of successful analyses, as witnessed for example by the following remark of Timothy Williamson:

Attempts to analyse the concepts means and causes, for example, have been no more successful than attempts to analyse the concept knows, succumbing to the same pattern of counterexamples and epicycles. The analysing concept does not merely fail to be the same as the concept to be analysed; it fails even to provide a necessary and sufficient condition for the latter. The pursuit of analyses is a degenerating research programme. (Williamson 2000, 31)

Chalmers and Jackson's response to this kind of objection is based precisely on considerations concerning scrutability. They stress that proposed definitions are usually found to be inadequate because they fall prey to counterexamples, i.e. hypothetical (or sometimes actual) cases with respect to which the purported equivalence between analysandum and analysans fails to hold. But to classify a case as a counterexample for a proposed analysis is simply to apply the general ability which is postulated in the scrutability theses. Consequently, the rejection of a proposed analysis due to a counterexample is itself based on conceptual analysis (cf. Chalmers \& Jackson 2001, 320ff.).

I agree with Chalmers and Jackson that the (alleged) fact that successful analyses of philosophically relevant expressions are nowhere to be found cannot in itself speak against conceptual analysis, for the reasons they mention. However, their defense of conceptual analysis only applies to the process of doing conceptual analysis (cf. chapter 1), since evaluating whether a given expression applies to a hypothetical scenario or not is hardly an end in itself. Any assessment of the value of conceptual analysis 
which does not also consider its potential aims and the prospects for achieving them would thus be incomplete.

In this chapter, I will therefore outline a number of goals which people have aimed (or might aim) at by doing conceptual analysis. In doing so, I will also try to clarify whether these ways of applying conceptual analysis to gain philosophical insights depend on explicit analyses, i.e. definitions, and if so, how. The second part of the chapter will deal with the question of whether it is realistic to expect that we can provide such definitions.

\subsection{The aims of conceptual analysis}

\subsubsection{Definitions - complete, partial, and absent}

Conceptual analysis as it has traditionally often been conceived and as it still is often conceived does aim at explicit analyses. Typically, such analyses provide necessary and sufficient conditions for the applicability of an expression in the form of a definition, as in 'bachelor $=_{\mathrm{df}}$ unmarried male adult human' or 'necessarily: $\mathrm{x}$ is a bachelor iff $\mathrm{x}$ is an unmarried male adult human'. This fits well with a conception of philosophy according to which it is (often) occupied with 'What is X?' questions. Here, the idea is that an analysis of the concept ' $X$ ' will also provide insight into the nature of X. But as should have become clear from preceding chapters, conceptual analysis, understood as an a priori enterprise, can only reveal the nature / metaphysical essence of $\mathrm{X}$ if the relevant term ' $\mathrm{X}$ ' is epistemically transparent: For other kinds of expressions, the metaphysical application conditions cannot be determined a priori.

In a case where we are interested in the nature of the referent of an expression which is not epistemically transparent, conceptual analysis can obviously not do the whole job. It may still have some role to play, though. Since an expression's primary intension determines its secondary intension (in a context), such an investigation should at least in some sense rely on our grasp of the relevant concept (cf. also my discussion on the role of primary intensions in determining the subject matter in 4.2). 
Nevertheless, it would be unreasonable to think that in order to reveal the nature of $X$ in a case where ' $X$ ' is epistemically opaque, we have to provide an explicit analysis of the expression. Lavoisier surely did not have an analysis of the term 'water' at hand when he discovered that water is $\mathrm{H}_{2} \mathrm{O}$. An implicit grasp of the concept (together with accurate sense perception) was sufficient for him to know that he was experimenting with samples of water.

Things are different if ' $\mathrm{X}$ ' is epistemically transparent. In such a case, conceptual analysis alone can reveal X's nature or essence (leaving aside worries about a possible divergence between a thing's necessary properties and its essence, cf. Fine 1994). But here, rather trivially, one does require an explicit analysis of ' $\mathrm{X}$ '.

In the remainder of this section, I will be concerned with the question of how conceptual analysis can be useful where it yields only partial definitions or no explicit analyses at all.

For many purposes, it can already be helpful to have a partial definition of an expression. This can mean, for instance, that one has managed to identify either one or more necessary conditions or just sufficient conditions for the applicability of the expression. Let me consider the latter case first:

Take a description of a thought experiment $\mathrm{D}_{\mathrm{TE}}$. If one (correctly) judges $\mathrm{D}_{\mathrm{TE}}$ to imply $\mathrm{X}$, then one has already identified sufficient conditions for the presence of $X$ - if ' $X$ ' is semantically neutral, then these are both epistemically and metaphysically sufficient conditions. Since the description of a thought experiment will usually comprise a large number of sentences invoking various kinds of facts, such sufficient conditions are not the sort of thing that usually enters into an analysis. Nevertheless, identifying them may provide important insights, for example into the relation between different kinds of properties. To illustrate: Take the phenomenal fact $\mathrm{P}$. If a physicalist could show that $\mathrm{P}$ is implied by a description of a scenario which only invokes physical (or, say, functional) facts, this would be a huge success. To take this idea one step further: If $P$ 
is a conjunction of all phenomenal facts and $D_{\text {Phy }}$ a (partial) description of the physical state of the world, then many would say that showing that $\mathrm{D}_{\text {Phy }}$ implies $\mathrm{P}$ amounts to establishing physicalism. This might even be so if there are no necessary physical conditions for the obtaining of any phenomenal facts to be had - in the case that the phenomenal facts could have also been realized by non-physical facts.

Accordingly, being able to identify sufficient conditions for the applicability of an expression, in whatever format, can be of major philosophical importance. This is not to say that finding less inclusive sufficient conditions is not generally preferable: For example, it would be good to know not only that phenomenal facts are entailed by physical facts, but which physical facts they are entailed by. ${ }^{144}$ In any case, we have thus already determined one potential use of conceptual analysis which does not depend on (complete) explicit analyses.

Similar considerations apply to cases where we can give necessary conditions for the applicability of an expression: To start with, when one has identified sufficient conditions for $\sim X$, then one can trivially extract necessary conditions for X. For example, when we have a description of a hypothetical scenario $D_{T E}$ to which ' $X$ ' does not apply, then $\sim D_{T E}$ is a necessary condition for $\mathrm{X}$. Such kinds of necessary conditions will typically not be very useful. However, this is not (merely) due to $\sim \mathrm{D}_{\mathrm{TE}}$ yielding a negative condition, as can be seen from the fact that many would consider it as an important insight that for a belief to count as knowledge, it must not be based on a falsehood. Rather, the problem is that $\mathrm{D}_{\mathrm{TE}}$ contains so many facts that little information is provided by the recognition that their conjunction has to be absent for ' $\mathrm{X}$ ' to be applicable.

In many cases, however, it is possible to determine informative and positive necessary conditions. Take the case of knowledge, which is generally considered as a standard example for the failure to provide

\footnotetext{
144 This may well be a merely theoretical distinction. It is plausible that in practice, it is not possible to show that $\mathrm{D}_{\text {Phy }}$ entails $\mathrm{P}$ without being more explicit about the kinds of physical facts which do so.
} 
explicit analyses. ${ }^{145}$ There is widespread consensus even among those who say that the term 'knowledge' cannot be defined that truth, and being believed, are necessary conditions for a proposition to be known. In the case where the expression in question is epistemically transparent, determining necessary application conditions can help to reveal various kinds of necessary truths, even in the absence of complete analyses. Where opaque expressions are concerned, being equipped with (epistemically) necessary conditions will still allow one to identify inferential connections between our concepts.

I just discussed how conceptual analysis can be valuable where it only delivers partial analyses. Let me end this section by briefly addressing how conceptual analysis could contribute to philosophical progress in the absence even of such partial analyses:

According to ordinary language philosophers, many philosophical problems can be resolved by considering the usage of relevant linguistic expressions. This kind of analysis need not lead to an explicit definition at any stage. As many of its advocates have an agenda that is quite alien to the account I have tried to defend, I do not want to delve too deeply into the details of ordinary language analysis. But still, there are ways to use conceptual analysis which are in the spirit of ordinary language philosophy and which are clearly compatible with two-dimensionalism. For example, one could examine the usage of an expression to resolve disputes which are merely verbal: If we discover that your evaluation of some key scenarios involving an expression which is relevant to our disagreement differs from mine, even if both of us have fully understood the case and thought carefully about it, this may suggest that the disagreement is just verbal. This kind of conceptual analysis only needs to assume scrutability and does not have to rely on any kind of explicit analysis.

${ }^{145}$ But see my discussion in 7.2 below. 


\subsubsection{Reductive explanations}

According to Joseph Levine, Chalmers and Jackson, and also Jaegwon $\mathrm{Kim}$, conceptual analysis plays a crucial role in reductive explanations (cf. Levine 1993; Chalmers \& Jackson 2001; Kim 2005, $111 \mathrm{ff}$.). The role of a priori scrutability in reductive explanations, in which higher-level phenomena are explained by invoking lower-level facts, was already briefly discussed in the previous chapter. There I also mentioned that Block and Stalnaker reject Chalmers and Jackson's claim that a reductive explanation requires that the higher-level facts are a priori entailed by the lower-level facts.

But assume that both the explanandum and the explanans are couched in epistemically transparent terms. Then, given what I have argued in the preceding chapters, it is clear that for a phenomenon to be reductively explainable, it has to be a priori entailed by the facts in the explanans. This is because the higher-level phenomenon has to supervene on the relevant low-level facts, i.e. it has to be metaphysically determined by them. For otherwise, there could be variation in the higher-level facts which is independent of what happens on the lower level, and thus there would at least be some aspect of the higher-level phenomenon which cannot be explained by these lower-level facts. And if, as we have assumed, only epistemically transparent expressions are used, metaphysical supervenience coincides with a priori supervenience.

Let me bring in another plausible constraint on reductive explanations: Given the lower-level facts, it must be intelligible/transparent to us why a particular higher-level fact obtains (cf. also chapter 6). To illustrate: Assume that $\mathrm{L}_{1}$ is a lower-level state and $\mathrm{H}_{1}$ is the corresponding higherlevel state, i.e., the higher-level state which obtains when the system in question is in $\mathrm{L}_{1}$. Now suppose further that from our (epistemic) perspective, $\mathrm{L}_{1}$ seems as compatible with $\mathrm{H}_{1}$ as with $\mathrm{H}_{2}$ or $\mathrm{H}_{3}$. In other words, $\mathrm{L}_{1}$ makes the occurrence of $\mathrm{H}_{1}$ just as likely as that of $\mathrm{H}_{2}$ or $\mathrm{H}_{3}$. In such a case, it hardly makes sense to say that $\mathrm{L}_{1}$ can explain the presence of $\mathrm{H}_{1}$. 
If one adds together what has just been argued, then it is natural to postulate the following two constraints on a successful reductive explanation of $\mathrm{H}$-facts on L-facts: a) the $\mathrm{H}$-facts must be a priori entailed by the L-facts; b) it has to be shown that a) obtains. ${ }^{146}$

Nevertheless, both of these constraints are contestable. Take a) first. The argument for a priori entailment was based on the premise that both the explanans and the explanandum are couched in epistemically transparent expressions. With respect to the explanans, this assumption seems reasonable: It would be strange to give an explanation using terms whose metaphysical application conditions are not transparent to us. However, it is hard to see why one would not want to explain phenomena which are described in opaque terms. As an example, take the disposition of water to form drops which was already mentioned in the previous chapter. One might want to explain this feature by drawing on facts about the behavior of aggregates of $\mathrm{H}_{2} \mathrm{O}$ molecules in certain conditions. But, since 'water' is epistemically opaque, it is not a priori that if $\mathrm{H}_{2} \mathrm{O}$ molecules behave in such-and-such a way, then water behaves in such-and-such a way. To draw this inference, one at least needs the additional empirical premise that water is $\mathrm{H}_{2} \mathrm{O}$.

There is a natural response to this objection: The fact that water is $\mathrm{H}_{2} \mathrm{O}$ is itself a priori entailed by low-level features. There is thus no need to invoke an additional empirical premise. But I think this response fails. I do agree that water's being $\mathrm{H}_{2} \mathrm{O}$ can be a priori derived from low-level facts - in fact this is just an instance of the a priori scrutability thesis (CJ++) (or simply of $\left(\mathrm{CJ}_{\mathrm{ap}}\right)$ which says that all truths about the actual world are a priori entailed by its canonical description). However, I do not believe that the identity of water and $\mathrm{H}_{2} \mathrm{O}$ can be a priori derived from the facts invoked in the explanans of the relevant reductive explanation. For consider what one would need in order to draw such an inference:

\footnotetext{
${ }^{146}$ Levine, Chalmers and Jackson, and Kim clearly endorse a). It is less clear whether they endorse b) as well. But in any case, their model of reductive explanation involves not only a priori derivability, but substantial parts of actual a priori derivations as well.
} 
In chapter $3 \mathrm{I}$ argued that it is a priori that water, if it exists, is the substance which plays a certain theoretical role, including its floating in our oceans and lakes, being drinkable, sometimes falling from the sky in drop shape and sometimes in flake shape, freezing at $0^{\circ}$ Celsius, etc. Now consider the vast amount of information one would need to determine that this theoretical role is played by $\mathrm{H}_{2} \mathrm{O}$ : Information about the distribution of $\mathrm{H}_{2} \mathrm{O}$ molecules on our planet and in its atmosphere, their behavior in various kinds of circumstances, their effects on our organisms, etc. It would be absurd to demand that a reductive explanation of the disposition of water to form drops has to invoke all of these facts.

One can of course insist that reductive explanation does require completely a priori entailments. One would then have to say that in the example I gave, one has really only explained the disposition of $\mathrm{H}_{2} \mathrm{O}$ molecules to form drops. But I do not see an independent reason for this claim. It seems perfectly natural to say that one has given a reductive explanation of a macroscopic feature of water, even if the explanation is not a priori through and through. We can be very confident that water is indeed $\mathrm{H}_{2} \mathrm{O}$, so it does not do any harm to use this piece of empirical knowledge as a premise in the reductive explanation.

If one accepts this result, one is forced to reject constraint $b$ ) as well - if the entailment from explanans to explanandum does not have to be purely a priori, then one cannot require that it is shown that there is such an entailment, either. Moreover, I would suspect that even in cases where the explanans does entail the explanandum a priori, it will often be practically impossible or at least excessively difficult to draw all the relevant a priori inferences. In many such cases, it may do, for instance, to work with approximate models or to invoke a well-established empirical premise at some point or other. a) and b) are thus arguably too strong as constraints on reductive explanations. Nevertheless, the point I made above about the need for intelligible/transparent connections between explanans and explanandum remains valid. And in general, the connection between an Lfact and an $\mathrm{H}$-fact will be intelligible if one can derive the $\mathrm{H}$-fact from the L-fact without additional empirical premises. This suggests that one should 
be parsimonious in invoking such empirical premises. In judging the adequacy of the explanation, one should also take into account at which part of the explanation these premises are invoked and which form they take. For example, it would seem inadequate if one had to postulate mediating 'bridging principles' at an important stage of the explanation which themselves remain unexplained.

Which kind of inquiry one should reserve the label 'reductive explanation' for is arguably not crucial. I already noted that if a phenomenon can be reductively explained (in a neutral sense of the term) by certain lower-level features, then either the properties of the higher-level phenomenon are a priori entailed by the lower-level features - in a case where only epistemically transparent expressions are used - or they are a priori entailed from a broader basis. Furthermore, in the latter case there is always an explanandum nearby which is in fact a priori entailed by the explanandum: In the example I gave above, for example, this would be the disposition of $\mathrm{H}_{2} \mathrm{O}$ to form drops. It is evident that if one could show how the facts in the explanandum are a priori entailed by those in the explanans, this would provide an excellent explanation. Given this, there is no reason why one should not take this ideal case as a model of explanation and try to come as close to it as possible.

In the following, I will have a closer look at how the type of a priori reductive explanation advocated by Levine and others is supposed to proceed. A key question which I will try to answer is whether such reductive explanations require explicit analyses, and if so, to what extent.

One reason why people have been hesitant to concede that conceptual analysis plays an important role in reductive explanations is their general skepticism regarding the possibility of giving explicit analyses (cf. e.g. Block \& Stalnaker 1999). In their joint paper, Chalmers and Jackson address this worry (cf. Chalmers \& Jackson 2001). They point out that their claim is only that the facts in the explanandum are a priori entailed by those in the explanans. This is compatible with the absence of a definition of the explanandum, just like it is possible to derive facts about knowledge from a 
description of a hypothetical case (for instance a Gettier scenario) in the absence of a definition of 'knowledge'. Chalmers and Jackson's case for the importance of conceptual analysis in reductive explanations is thus based on scrutability, rather than on explicit analyses. On the face of it, the ability mentioned by a thesis such as $(\mathrm{CJ}++)$ seems tailor-made for a reductive explanation: Take a higher-level fact $\mathrm{H}$ and a lower-level fact $\mathrm{L}$. If it can be shown that $\mathrm{H}$ is a priori entailed by $\mathrm{L}$, then this offers a perfect explanation for the occurrence of $\mathrm{H}$ in the sense outlined above: Given $\mathrm{L}$, it becomes intelligible why H occurred, or even had to occur. Does this mean that reductive explanations can just rely on scrutability and do not need any kind of explicit analyses? As I will show in the following, this is far from clear.

The way I put things so far might convey the impression that the process of giving a reductive explanation is completely bottom-up. But this cannot be correct. First of all, it would be absurd to think that we start from complete low-level information and then reason our way upwards, deriving one higher-level truth after the other until we stumble upon our H. Furthermore, and somewhat less trivially, one does not typically aim at a reductive explanation of a singular fact, event or state. Rather, reductive explanations target wholesale phenomena. That is to say, firstly, that one deals with a type of fact (event, state) which usually has a huge number of instances. (Arguably, one may at least occasionally want to explain a range of merely possible instances as well.) Secondly, the target of the explanation will typically comprise a number of different configurations or states at the macro-level.

Consequently, in order to give a reductive explanation it will not suffice to derive one higher-level fact from a lower-level fact - if only because one has thereby at best explained one kind of high-level configuration. But needless to say, we cannot derive every single instance of the relevant higher-level fact(s) from lower-level facts, either. So if the whole process was indeed bottom-up and we had to rely on our ability to derive higherlevel facts from lower-level facts alone, we would not be able to explain the whole phenomenon. 
If one takes the scrutability thesis and our corresponding ability as the sole basis of reductive explanation, one thus seems at a loss to say how such an explanation is supposed to work. For this reason, one will at least have to make an additional assumption to the effect that we have some kind of prior grasp of the explanandum, beyond our ability to recognize its instances if confronted with lower-level information. It is thus no accident that when people outline how a reductive explanation which is based on a priori entailments proceeds, they usually start with a definition of the explanandum (cf. e.g. Levine 1993, 131). Let me briefly sketch how such a reductive explanation could proceed:

Suppose one aims to give a reductive explanation of heat. In the first step, one will provide an analysis of 'heat', in the sense of explicating its primary intension. Plausibly, this will mean identifying the causal role associated with the term: Heat is the phenomenon which makes ice melt and water boil, which brings about tissue damage, causes gases to expand, etc.

In the second step, one identifies the occupant of this causal role: Mean molecular kinetic energy (or in the cases I mentioned (relatively) high mean molecular kinetic energy) is what does all these things. The determination of the occupant of the theoretical role is enabled by the fact that once we suspect that the relevant effects might be related to the energy-level of molecules, we can reason from facts in the explanans to what we actually observe at the macro-level. Here is how this might look, in very crude form: When the mean molecular kinetic energy of $\mathrm{H}_{2} \mathrm{O}$ molecules reaches a certain level, the ordering of the molecules in the crystalline structure (of ice) will collapse and the aggregate phase of the sample will change from solid to liquid. If one increases the energy level even further, one thereby increases the likelihood of the molecules to escape the liquid and thereby the liquid's vapor pressure, up to the point where the vapor pressure will equal the pressure of the surrounding environment and thus vapor bubbles will start to form in the liquid. When proteins in our skin interact with high-level particles, they will disintegrate, resulting in tissue-damage. And when the molecules in a gas gain 
molecular kinetic energy, the mean distance between them will increase which means that the gas expands. ${ }^{147}$

We just saw that if one starts from a definition of the explanandum, one can at least tell an intelligible story of how the envisaged explanation could proceed. This does not mean that reductive explanations have to begin with an analysis of the phenomenon to be explained, however. It may usually be sufficient to give an approximate characterization of the explanandum, or even just to rely on our implicit grasp of it. And arguably, something less than a complete definition typically has to be sufficient. For, it is very likely that for most phenomena which have been reductively explained, no analysis which holds up to philosophical standards - such as, say, a definition which is not prone to counterexamples - has been presented.

Accordingly, I do not want to deny that it is possible to provide reductive explanations in the absence of perfect definitions. Yet, let me note once more that such an explanation depends on more than bottom-up scrutability; we also need the ability to overlook what the phenomenon in question comprises. In particular, we need to be able to assess not only where to look, but also when we have explained everything which needed to be explained. The latter point can be illustrated by drawing an analogy with what happened in the case of knowledge. Based on their understanding of the term, people thought for a long time that having a justified true belief is really all there is to knowing something. But they missed a relevant aspect of the phenomenon, even after considerable reflection about their subject matter. Something similar could happen in the case of reductive explanations. Unless one has a complete (and correct) definition of the explanandum, it might happen that one believes to have provided a reductive explanation even though there is an important dimension to it which is yet to be accounted for.

David Lewis' account of pain might serve as an example (cf. Lewis 1983): In Lewis' view, pain can be defined in functional terms. ${ }^{148}$ Let us thus say

${ }^{147}$ If one adds to this a third step in which heat is identified with mean molecular kinetic energy, then the whole process follows quite closely the general model of reduction in the Canberra Plan, which will be discussed in the next section. 
that pain is the phenomenon which causes avoidance behavior, which brings people to utter things like 'Ouch!' or 'That hurts', which makes them consult a doctor or take a pill, etc. If this definition is correct, then it is plausible that pain can be reductively explained in purely physical terms, since the relevant functional roles are plausibly played by broadly physical features. However, critics have objected that Lewis' account of pain leaves out a crucial aspect of pain, namely the distinctive phenomenal quality associated with it. They say that since there cannot be a definition of pain in functional terms, there is no way to give a reductive explanation of pain in physical terms (cf. e.g. Levine 1993; Chalmers 1996; Kim 2005). If these critics are correct, an analysis in the spirit of Lewis might still capture many or even most important aspects of pain and thus allow a reductive explanation of these aspects in physical terms. Nevertheless, the overall explanatory project will be doomed to failure.

The example suggests that even if in many cases, a "rough-and-ready analysis" of the explanandum (Chalmers 1996, 43) or even our implicit grasp of it turns out to be sufficient for giving a reductive explanation of a phenomenon, this method is not fool-proof: Unless one has a complete and correct definition at hand, it can happen that one misses relevant aspects which need to be explained.

\subsubsection{The Canberra Plan}

The Canberra Plan is intimately linked with a specific account of the objectives of metaphysics. Before I turn to a discussion of the Canberra Plan itself, let me thus outline this account which has been most prominently advocated by Lewis and Jackson.

In Jackson's words, 'serious metaphysics' does not occupy itself with enumerating everything there is in the sense of drawing up a huge list of

\footnotetext{
148 With the addition that pain only needs to play this functional role usually in members of the relevant species, in order to account for occurrences of pain which do not play the functional role ('mad pain') and states which have this role but are not pain ('Martian pain').
} 
things which includes black holes, electrons and nucleic acid, as well as spoons, potato chips and billiard cues. Rather, it seeks to compile a much more parsimonious account in which only the fundamental building blocks of the world are mentioned (cf. Jackson 1998a, ch. 1). On Jackson's own account, for instance, this will be a description in terms of a completed physics.

However, this idea immediately raises a problem. In the more parsimonious fundamental description of the world, spoons, potato chips or billiard cues will hardly appear - after all, the whole idea of doing 'serious metaphysics' was to provide a shorter list. But at the same time, the list should still be comprehensive. So does this mean that one has to say that there are no shoes, potato chips or billiard cues? This would obviously be quite an unhappy result. The solution to the problem is based on the idea that many kinds of facts which are not explicitly mentioned in the fundamental description of the world nevertheless appear implicitly in it. The facts which appear implicitly in the description are those which are metaphysically entailed by it, i.e. those which supervene on the distribution of fundamental properties.

At this point, another important task of metaphysics becomes apparent: It aims to show how the fundamental description of the world makes the nonfundamental facts true. Metaphysics thus deals with 'location problems' (this is again Jackson's expression), i.e. it tries to locate higher-level phenomena like spoons and potato chips or, more realistically, knowledge, meaning and actions in the basic physical reality. Given my discussions in the preceding part of this work, it should not come as a surprise that conceptual analysis plays an essential role in this kind of enterprise. The key idea here is that one aims to show that higher-order facts are metaphysically entailed by the fundamental facts by pointing out how they are a priori entailed by them. The theoretical underpinning for this kind of procedure is provided by $(\mathrm{CJ}++)$, and more generally by the thesis of metaphysical plenitude: Provided that the fundamental description of the world is couched in purely epistemically neutral vocabulary, every fact 
which is epistemically entailed by this description is also a priori entailed by it and vice versa. ${ }^{149}$

Just like in the case of reductive explanations, the question arises how this objective is supposed to be achieved. As I already noted in the previous section, one cannot just start from a complete fundamental description and hope to derive specific higher-order truths from it. This may be the reason why the starting point of the Canberra Plan is at the top level: Its first step consists in an analysis of the relevant higher-level phenomenon. In what follows, I will set forth the specifics of the Canberra Plan in some more detail.

The first step of the Canberra Plan is based on Lewis' account of defining theoretical terms which is in turn based on the work of Frank Ramsey and Carnap (cf. Lewis 1970): Suppose you have a theory $T$ about a specific domain. $T$ comprises some theoretical terms $t_{1}, \ldots, t_{n}$. Of course, there are also many other kinds of terms which figure in $\mathrm{T}$. Lewis calls these the Oterms (here, the ' $\mathrm{O}$ ' stands for 'Other'). One may assume that the theoretical terms are newly introduced with the theory and derive their interpretation only from their relation to the O-terms as specified in T. Now the theoretical terms are replaced by free variables, thus yielding $T\left(x_{1}, \ldots\right.$, $\mathrm{x}_{\mathrm{n}}$ ). Lewis calls this the realization formula of $\mathrm{T}$ : Any $\mathrm{n}$-tuple of entities which satisfies the formula realizes $\mathrm{T}$.

The Ramsey sentence of $\mathrm{T}$ says that $\mathrm{T}$ has such a realization: $\exists \mathrm{x}_{1}, \ldots, \mathrm{x}_{\mathrm{n}} \mathrm{T}$ $\left(\mathrm{x}_{1}, \ldots, \mathrm{x}_{\mathrm{n}}\right)$. And its Carnap sentence says that if $\mathrm{T}$ is realized, then the theory's postulate is satisfied: $\exists x_{1}, \ldots, x_{n} T\left(x_{1}, \ldots, x_{n}\right) \rightarrow T\left(t_{1}, \ldots, t_{n}\right)$.

Importantly, the Carnap sentence is supposed to be analytic, i.e. it is analytic that if the realization formula of $\mathrm{T}$ is satisfied, then the theory is correct. According to Lewis, the same goes for the inverse of the Carnap sentence: $\sim \exists \mathrm{x}_{1}, \ldots, \mathrm{x}_{\mathrm{n}} \mathrm{T}\left(\mathrm{x}_{1}, \ldots, \mathrm{x}_{\mathrm{n}}\right) \rightarrow \sim \mathrm{T}\left(\mathrm{t}_{1}, \ldots, \mathrm{t}_{\mathrm{n}}\right)$. This implies that the theory $\mathrm{T}$, i.e. its postulate, is a priori (analytically) equivalent to its Ramsey sentence. Note also that none of the T-terms figures in the Ramsey

${ }^{149}$ As was already noted a couple of times, to be able to derive all truths one will additionally need indexical information and a clause which states that the description is complete. 
sentence. The Ramsey sentence thus provides a way of dispensing with the T-terms; it is surely no stretch to say that it thus provides an analysis of the T-terms.

The fact that the theoretical terms are replaced by variables suggests that Lewis' model only applies to names. However, in his view predicates are eligible as well; they simply have to be treated as names of properties. For example, if 'green' is the relevant T-term, then ' $x$ is green' is rephrased as something like ' $x$ has greenness', where 'has' is an O-term. Furthermore, Lewis' theory places no constraints on what one can consider as a theoretical term in a given context. Thus in principle, any kind of term or set of terms can be subject to this kind of analysis. For this reason, Lewis' account of defining theoretical terms can serve as the basis of a comprehensive metaphysical project of reduction.

Here is how this project is supposed to proceed: One starts with an (alleged) higher-order phenomenon. In a first step, one constructs a Ramsey sentence, with those terms which are relevant for characterizing the phenomenon figuring as T-terms. One thereby gets an account of the relations of that phenomenon to other kinds of features, as described by the O-terms; call these relations the T-role. Then, one goes about determining what, if anything, in the fundamental description satisfies the T-role. If one succeeds, one has thereby located the higher-level phenomenon within the fundamental description of the world. Given this, one can also reason upward from the fundamental description to arbitrary truths about the phenomenon in question, in accordance with (CJ++).

Let me flesh out this highly abstract picture with the aid of an example. Suppose the higher-order phenomenon to be reduced is water. 'Water' can thus be considered as the sole theoretical term. The Ramsey sentence will roughly say something like this: There is an $\mathrm{x}$ such that $\mathrm{x}$ actually stands in an acquaintance relation to me, is clear and drinkable, sometimes falls from the sky in drop shape, expands when it freezes, ...; call this the water role. ${ }^{150}$ Now one tries to determine what plays this theoretical role: Since,

$\overline{{ }^{150} \text { Cf. 3.1.1 for more details. }}$. 
as we all know, $\mathrm{H}_{2} \mathrm{O}$ plays this theoretical role, one can conclude that water is $\mathrm{H}_{2} \mathrm{O}{ }^{151}$

In the following, I will discuss a couple of issues concerning the commitments of the Canberra Plan, its viability and its potential merits for philosophy. I will first, i.e. in 7.1.3.1, say a few words to clarify how Ramsey sentences are built and how this relates to the problem of ensuring unique reference. Then, in 7.1.3.2, I will discuss two worries regarding the scope of the Canberra Plan and finally, in 7.1.3.3, two further worries concerning its practical feasibility.

\subsubsection{Ramsey sentences, primary intensions and unique reference}

Let me start by looking in a bit more detail at how the relevant Ramsey sentences are supposed to be constructed. In Psychological and Theoretical Identifications, Lewis expresses the view that one has to start with a collection of platitudes about the subject matter at hand (cf. Lewis 1972, 256). ${ }^{152}$ Jackson occasionally comments in the same vein, for instance in the context of a reduction of moral properties (cf. e.g. Jackson 1998a, 130). The example of water I gave above seems to confirm this idea. The Ramsey sentence I sketched there mainly (but really only mainly) looks like a collection of platitudes about water. One should not assume, however, that such a procedure is generally adequate. To see this, recall that the Ramsey sentence is supposed to provide a definition of the term(s) in question. In effect, it should thus spell out its/their primary intension(s). But as I argued most extensively in chapter 3, one important lesson to be drawn from Kripke's arguments against descriptivism is that the properties associated

\footnotetext{
${ }^{151} \mathrm{H}_{2} \mathrm{O}$ is most likely not included in a fundamental description of the world, but it is at least sufficiently low-level to illustrate the general schema. The underlying problem that there can still be many levels between what is said in the Ramsey sentence and in the fundamental description will be addressed in 7.1.3.3.

152 The subject matter which he was concerned with there was folk psychology. He later changed his mind, though, cf. Lewis 1994, 216.
} 
with an expression do not always correspond to what readily comes to a subject's mind when she comes across a token of this expression.

From what I just said it can be concluded that the question of how one should go about constructing Ramsey sentences amounts to the question of how one can provide definitions. I will leave a discussion of this difficult issue to 7.2 .

It has been argued that Ramsey-style analyses are liable to the so-called 'permutation problem' (cf. Smith 1994, 48ff.): Suppose that the network of relations between T-terms and O-terms which we manage to identify is not sufficiently tight. Then it can happen that the resulting Ramsey sentence is satisfied by many (tuples of) entities; it does not permit us to uniquely locate the target phenomenon in the fundamental description.

The permutation problem is closely related to Kripke's argument from Ignorance against descriptivism which was discussed in chapter 3 . According to this argument, the reference of names cannot be determined by speaker associations because these associations are often too unspecific to yield a unique referent. The connection between the argument from Ignorance and the permutation problem is due to the fact that, as I just argued, the Ramsey sentence is supposed to reflect the primary intension(s) of the expression(s) in question, in other words the associated properties. The reply to Kripke's argument I gave in chapter 3 and my general defense of the idea that speaker associations determine reference with respect to every world considered as actual throughout much of this work thus apply to the permutation problem as well. Consequently, if the Ramsey sentence does not suffice for determining the reference of the T-term(s), this can only be due to the fact that it does not capture its/their primary intension(s) completely.

Nevertheless, it can of course happen that even a complete Ramsey sentence does not uniquely determine an entity or tuple of entities. We hardly have an a priori guarantee that, for example, our 'water theory' picks out exactly one substance. For this reason, the problem of what should be done when there is more than one realizer or tuple of realizers cannot be 
completely avoided. When Lewis introduced his account of defining theoretical terms (in How to Define Theoretical Terms), he argued that it is part of a theory that its postulate is satisfied by a unique tuple of entities (cf. Lewis 1970, 432ff.). Accordingly, if there is more than one tuple of entities which satisfies the postulate, this means that the theory is false and thus the T-terms fail to refer. In later work, Lewis seemed less sure how to deal with such cases, wavering between reference failure and indeterminacy (cf. Lewis 1999, 347). Wolfgang Schwarz argues, however, that the latter choice is clearly the appropriate one (cf. Schwarz 2009, 221). While I tend to agree with Schwarz, I do not think that this choice should be made on the level of our meta-theory. Rather, it should be decided by what we associate with the term or terms in question. There is, for instance, nothing which prohibits using a term in a way such that it refers only if it refers uniquely. In such a case, the relevant condition will be part of the term's primary intension and thus a clause to that effect will appear in its Ramsey sentence, as in 'There is exactly one x such that ...'.

\subsubsection{The scope of the Canberra Plan}

It is clear that one cannot 'ramsify away' all kinds of terms. One will always need some set of O-terms which define the T-terms. ${ }^{153}$ Since the general project is to locate higher-level phenomena in a fundamental description of the world, it is natural to think that the ultimately remaining O-terms will be those which are required to describe the fundamental properties and their distribution. ${ }^{154}$ However, Schwarz argues that there are terms which clearly seem unnecessary in a fundamental description, but which are nevertheless not eligible for a Ramsey-style analysis (cf. Schwarz 2009, 225ff.): Recall that in Lewis' theory, predicates are also replaced by variables in a Ramsey sentence - after having been rephrased

\footnotetext{
${ }^{153}$ In Lewis 1984, he defends a view which can be interpreted as leaving only one such O-term, namely 'naturalness'.

${ }^{154}$ Chalmers forthcoming discusses in detail what kind of vocabulary will have to be considered as primitive.
} 
as names for properties. But as Schwarz notes, there does not have to be an entity, such as a property, corresponding to a given predicate. An example he gives is causation: In Lewis' own theory of causation, absences can be causes. Accordingly, there is no causation relation, since absences are hardly eligible as relata. ${ }^{155}$ If one constructs a Ramsey sentence for 'causes', one will thus find that there is no entity which satisfies it. However, it would clearly be wrong to conclude from this that Lewis should be an eliminativist about causation. For in his theory, facts about causation are made true by lower-level facts. Consequently, Lewis' method of defining theoretical terms cannot be applied to every kind of expression. It is not obvious that this result is detrimental to the overall reduction project, however. One could still apply a very similar procedure in order to locate causation facts in a fundamental description: One starts with a conceptual analysis, for instance by systematizing the way the term 'causes' is applied to various hypothetical scenarios. Ideally, one will end up with an explicit analysis, yielding a way to express facts about causation without mentioning the term 'causes'. Then one can go about determining what in our fundamental description makes these facts true.

The next worry concerning the scope of the Canberra Plan is also due to Schwarz (cf. Schwarz 2009, 222-224). To elucidate what he aims at, let me remind you of a feature of many expressions which I discussed in chapter 5: There I argued that many of our terms are functional terms. Their purpose is not to pick out a specific individual, but rather to characterize a function, such that anything which has that function falls under the term. Now, Schwarz claims that for such expressions (he talks more generally about role terms), the second and the third step of the Canberra Plan, i.e. those steps in which the higher-order phenomenon is located in the fundamental description, are not applicable or at least irrelevant. Take his example of the property of being a clock: Suppose we have a Ramsey sentence for that property. According to the Canberra Plan, we are now supposed to locate it in our fundamental picture of the world. But it is

${ }^{155}$ This is remarked by Lewis himself, for instance in Lewis 2004. 
highly implausible that the various things which count as clocks have any kind of, say, microphysical commonality. We could describe the microphysical state of every existing clock and say that the property we are after is the disjunction of all of these microphysical states. However, that will not work. For what if someone would build another kind of clock? There are arguably even possible worlds containing clocks which are made of non-physical stuff. Schwarz concludes that the property we are after is just a (higher-level) functional property. Once we have the Ramsey sentence which spells out this function, we already have everything we could be looking for. It is thus neither possible nor necessary to follow up with a microphysical reduction.

While I agree with much of Schwarz's reasoning here, I do not agree with the conclusions he draws. First of all, it is true that we will not be able to give a microphysical reduction of the property of being a clock. But why should this be necessary, within Lewis' own account, for a successful reduction? For note that other possible worlds are part of his fundamental ontology as well. Lewis could thus say that the property of being a clock can be reduced to the set of microphysical or otherwise fundamental configurations constituting clocks across all possible worlds. This is in line with Lewis' general account of properties which he construes as sets of possible worlds.

Even more importantly, Schwarz' conclusion that the only step of interest in such cases is the first one of developing the Ramsey sentence is clearly exaggerated. Take a term which is mentioned by him as well, 'freedom'. Arguably, the term satisfies Schwarz' criteria for role terms: Free actions or choices (if there are any) will hardly share any microphysical commonality. And building the disjunction of microphysical realizations will not help either, since there could be different microphysical, or even non-physical, configurations which also constitute free actions or choices. Nevertheless, we might be interested in determining whether freedom does have a realizer on the level of fundamental properties, in order to find out whether there are free actions or choices. Alternatively, we might want to know whether it has, or could have, a physical realizer. Similar considerations 
apply to many other philosophically relevant terms which are role terms in Schwarz' sense as well, for example 'belief', 'pain', 'good', 'true', etc. Sometimes we are interested in knowing whether a higher-level role is realized by the fundamental properties, in order to know that the phenomenon in question exists. Alternatively, we may want to know whether it could be realized by what we take to be the fundamental properties. At other times, we are interested in whether the phenomenon could be realized by specific kinds of fundamental properties, for example by physical properties - either to locate the higher-level phenomenon, or to eliminate it, or to expand the repertoire of fundamental properties we posit. Accordingly, even in cases where we are dealing with role terms, the task of relating the corresponding properties with fundamental properties (in accordance with the Canberra Plan) will often be of philosophical interest.

\subsubsection{The practicability of the Canberra Plan}

The idea of locating ordinary macroscopic phenomena in a description of the world which only spells out the distribution of its fundamental properties should strike one as extremely ambitious. It is far from obvious how such a project should proceed and whether it can be successfully carried out. In the following, I will therefore identify the requirements of the Canberra Plan and evaluate its practical feasibility.

To begin with, the Canberra Plan relies on explicit analyses in the form of Ramsey sentences. One may wonder whether these analyses have to be complete and completely accurate, like in the case of reductive analyses discussed above. Plausibly, one will reach a verdict here which is similar to the one I reached there: In some cases, reasonably good though imperfect analyses will be sufficient. But in many cases, details clearly matter. To take just one example, consider Lewis' reduction of dispositions to counterfactual conditionals (cf. Lewis 1997). There is widespread agreement that there is a strong link between dispositions and counterfactuals. But many people think that there are counterexamples to each of the analyses Lewis has proposed; and this in turn has led many to 
believe that dispositions cannot be reduced to counterfactual conditionals at all. It is thus plausible that successful reductions in line with the Canberra Plan will often require explicit, complete and accurate analyses.

If one has constructed the relevant Ramsey sentence, the next task is to use this analysis to locate the target phenomenon in a fundamental picture of the world. It is not obvious how this can be done. For a start, an opponent of the Canberra Plan might claim that since we do not have a description of the distribution of fundamental properties at hand, we can hardly locate anything in it. In principle, that objection is surely correct. But at least in most cases we do not need such a complete description in order to solve a location problem. What we do usually need, though, is a rough grasp of what the fundamental properties will be like. Here, we have to rely on the assumption that our best scientific and/or philosophical theories are at least on the right track. If this assumption turns out to be wrong, then many of our putative reductions will be inaccurate. But this is a problem which cannot be avoided when one is concerned with metaphysics, so it is hardly a specific problem for the Canberra Plan.

Next, assume that we do have both a Ramsey-style analysis of our target phenomenon and a sufficient understanding of the fundamental properties. If we are lucky, then the O-terms will be part of the vocabulary used in the fundamental description of the world. In this case, the reduction is already complete. But since this is not to be expected, we are still confronted with a location problem: The problem of relating facts expressed by O-terms to those expressed by the fundamental vocabulary. In many cases, that problem will still not be trivial. To give an example: Lewis analyzes meanings in terms of linguistic conventions. Prima facie, it seems no more obvious how linguistic conventions relate to the fundamental properties than how meanings do. It is therefore reasonable to introduce a few more intermediate steps. Here is a crude sketch of what such a reduction could look like within Lewis' framework: Conventions are (very roughly) reduced to dispositions of speakers ${ }^{156}$ which are then analyzed via

${ }^{156}$ A bit more precisely, they are reduced to dispositions and intentional states of speakers. Since on Lewis' functionalist account, intentional states are themselves, by 
counterfactual conditionals. Counterfactual conditionals are analyzed in terms of relations between possible worlds; these (the relations, not the worlds!) are supposed to be reducible to qualitative similarities in the distribution of fundamental properties of these worlds and laws of nature. Laws of nature are in turn reduced to regularities among the fundamental (in particular, microphysical) properties. At each of these steps, one will require an analysis; each time, some of the former O-terms become T-terms and are thus defined away in the new analysis. And once again, when the reduction is complete, one can reason bottom-up, from the distribution of fundamental properties to the laws of nature to ... to meaning facts.

The Canberra Plan, as it is paradigmatically carried out by Lewis, is certainly a fascinating project. The idea to reduce everything there is to a limited basis of fundamental facts, moreover in such a way that it becomes a priori intelligible how the higher-order facts are grounded in the fundamental ones, seems highly appealing. At the same time, as I already mentioned above, the project is extremely ambitious and therefore also highly vulnerable. In the example I just gave concerning a possible reduction of meaning facts to fundamental properties, each of the steps involved is as intricate as it is controversial.

Nonetheless, analyses in the spirit of the Canberra Plan can be useful even for those who do not have such far-reaching reductive ambitions. If, for instance, one managed to establish just one of the steps I sketched of a Lewisian reduction of meaning, this would already be a major success. Generally speaking, one does not always have to reduce the target phenomenon to fundamental properties. One may just aim at showing that facts of a domain $\mathrm{X}$ are reducible to facts of domain $\mathrm{Y}$, or just to facts outside of domain $\mathrm{X}$ (for instance in the case of moral or generally normative facts). Or, maybe even more modestly, one just tries to show that all the facts about a specific domain can be expressed without using the

and large, reducible to dispositions, the statement that conventions are reduced to speaker dispositions is nevertheless approximately adequate. 
vocabulary which is considered to be characteristic for that domain, in order to dispense with these expressions.

The Canberra Plan thus offers an attractive model of how conceptual analysis could be done in philosophical practice. However, just like a number of other varieties of conceptual analysis which I have sketched in this chapter, it has to rely at least to some extent on the possibility of providing explicit analyses. In the following, I will turn to a discussion of the problems and prospects of this project.

\subsection{The trouble with definitions}

In the previous part of this chapter, I pointed out that conceptual analysis can be valuable even if it does not yield explicit analyses. However, in my discussion it also transpired that many ways in which conceptual analysis can be applied to gain philosophical insights do involve explicit analyses at one stage or other. For example, they are crucially involved in an attempt to reveal the essences of philosophically relevant categories, in the Canberra Plan of locating higher-level phenomena among the fundamental properties, and, as I have argued, they are at least sometimes also required in reductive explanations.

This dependence on explicit analyses will presumably seem worrisome to many. For it is often held that the history of attempted definitions of philosophical terms is a history of failure. It should be well worth considering whether the prospects of the project of providing definitions are really that bleak, given its implications for the scope and the potential value of conceptual analysis. The rest of this chapter will therefore be dedicated to that question.

\subsubsection{Adequacy conditions for definitions}

In order to evaluate the prospects of finding definitions, one will first have to determine the conditions which an adequate definition has to meet. It seems natural to demand that the definiens has the same application 
conditions as the definiendum, where application conditions are here to be understood as primary application conditions. That is to say, the primary intensions need to be identical. However, one could argue that this requirement is a bit too strict. One might for example hold that it is even a virtue of a definition if it reduces vagueness. In such a case the definiens has a definite extension in at least one scenario which is borderline according to the term to be defined. It may thus make sense to loosen the criterion just proposed slightly and require instead that the definiens should have the same primary application conditions with respect to cases where the definiendum has a definite extension.

Even that much is not uncontroversial. There may be cases where the expression to be analyzed needs to be slightly 'adjusted' for some reason or other. For example, the term in question might be inherently confused or just contingently empty. Alternatively, it may turn out that as it is, the term is of no particular philosophical use for other kinds of reasons. In each of these cases, it could be useful to offer an analysis of the original term with at least slightly different application conditions. The analysis might thereby yield an expression which is not confused (and thus not necessarily empty), not empty or just of greater philosophical interest for another reason. Such an approach seems well in line with Lewis' views and also with Jackson's. Jackson explicitly says that often, conceptual analysis will involve a slight departure from the folk concept at hand (cf. Jackson 1998a, 44-46). As an example, he discusses what it takes for an action to be free: Jackson believes that (our folk concept of) a free action is strictly speaking not compatible with determinism:

What compatibilist arguments show, or so it seems to me, is not that free action as understood by the folk is compatible with determinism, but that free action on a conception near enough to the folk's to be regarded as a natural extension of it, and which does the theoretical job we folk give the concept of free action in adjudicating questions of moral responsibility and punishment, and in governing our attitudes to the questions of those around us, is compatible with determinism. (Jackson 1998a, 44f.) 
Accordingly, if the compatibilist offers an analysis of 'free action', the analysans will have application conditions which differ at least slightly from those of the analysandum.

In my view, such an adjustment of the expression to be analyzed is not unproblematic. A skeptic concerning free action will say that if Jackson's assessment of the situation is accurate, then she wins the debate. For in this case, it may well be true that there are actions that fall under a notion which is in some sense similar to our notion of free action, but nevertheless, there are no free actions. ${ }^{157}$ As another example, consider the question of whether there are moral properties. John Mackie argues that there are no such things as categorical imperatives, i.e. there are no judgments which in themselves give us a reason to act / motivate us to act. His reason for holding this view is that he thinks there are no properties which are intrinsically motivational. Furthermore, Mackie claims that our moral discourse presupposes that there are such 'objectively prescriptive' properties. For this reason, he considers this discourse to be fundamentally flawed (cf. Mackie 1977): It presupposes the existence of moral properties which do not, or even cannot, exist.

Now suppose one goes about defining moral terms, proceeding as follows: First one slightly adjusts our folk terms such that it is no longer a necessary condition that knowledge of their extension in itself motivates a subject to act. Then one develops a definition in terms of, say, naturalistically acceptable properties. It should be obvious that one has not thereby shown that there are naturalistically acceptable moral properties. Quite the opposite: If our moral terms are such that their applicability requires the existence of intrinsically motivating properties and if moreover there are no such properties, then, rather trivially, our moral terms are empty, regardless

\footnotetext{
${ }^{157}$ Since our world is most likely not deterministic, this does not follow immediately. However, it is hard to see how the falsity of determinism is supposed to help the realist about free actions, so the skeptic's conclusion may nevertheless be justified. In this case, one could say that if Jackson's assessment is accurate, then there are necessarily no free actions.
} 
of whether there are non-empty terms in the vicinity which might even be philosophically useful.

This does not mean, however, that I consider Jackson's view to be fundamentally flawed. I agree with him that the concepts we find in a natural language may not always pick out philosophically interesting categories or, more generally, that they may not always be perfectly suitable for philosophical purposes. In many such cases, it can be useful to replace a concept with one which has similar application conditions: The successor may well help to clarify important philosophical questions, for instance. The examples I just gave show, however, that one has to be careful in drawing philosophical conclusions from such analyses. At bottom, this is because philosophical questions are phrased in terms of a natural language; these folk terms thus define our subject matter (cf. 4.1). It is natural to liken the kind of 'adjusting analysis' proposed by Jackson to Carnapian explications (cf. Carnap 1947/1956). Jackson's remarks do suggest that there are contexts in which explications are sufficient for our purposes. Given what I have just argued, however, it is important to distinguish these from actual definitions. Let me thus extract the following minimal condition of adequacy from the previous considerations: In a successful definition, the application conditions of the definiens have to be similar enough to those of the definiendum to not beg any philosophically relevant questions which might come up.

Let me add the following, rather trivial condition of adequacy for definitions to the one just identified: They must not be of the form ' $a=a$ ', i.e. the definiens must use different expressions than the definiendum. Ideally, the terms used in the definiens or the features expressed by them will in some sense be more basic, but this should not be considered necessary. Often, definitions are also expected to be short, as in 'bachelor $={ }_{\mathrm{df}}$ unmarried male adult human' or 'human $=_{\mathrm{df}}$ rational animal'. However, I see no reason to take this as a necessary condition, either. One could require that definitions are of manageable length. However, depending on the context (and maybe a subject's cognitive capacities), analyses which 
are much more extensive than anything philosophers have so far come up with can still be manageable.

I conclude that the task we are confronted with in constructing a definition of a given term amounts to that of finding a string of other words of manageable length yielding an expression with at least close to identical primary application conditions.

\subsubsection{Objections to the eligibility of definitions}

\subsubsection{Objections from the relation between definiendum and everything else}

In what follows, I will identify a number of necessary conditions for the feasibility of the task I just outlined. All of these conditions concern the relation of the definiendum with those expressions which could be used in the definiens, or alternatively with the facts expressed by them. I thereby provide skeptics of the project of giving definitions with targets for potential objections, since they could deny that these conditions are met by philosophically relevant expressions. My discussion will show, however, that there are reasons to believe that the conditions in question obtain in the case of most of the expressions with which we are concerned in philosophical practice.

Let me call the expression to be defined $\mathrm{E}$ and the properties denoted by it the E-properties. Now suppose that the E-properties do not supervene on any other properties, i.e. they are fundamental. In such a case, E can only be defined if it is epistemically opaque. Otherwise, it has to be considered as primitive. We have thus identified one necessary condition for the definability of E: E must not transparently denote properties which are metaphysically fundamental.

Next, assume that E-properties supervene on other kinds of properties for which, however, we do not have concepts. E-properties are thus not 
metaphysically fundamental, but one could say that $\mathrm{E}$ is intentionally fundamental. With respect to the issue at hand, the implication is the same: Such a term would be primitive and thus unanalyzable. The second necessary condition for the definability of an expression is therefore this: The definiendum must supervene on properties to which we have intentional access, i.e. it must not be intentionally fundamental.

Now let me outline a third and final way in which E could be primitive: It could happen that we have intentional access to properties on which Eproperties supervene, yet lack the linguistic means to express these properties. E-properties could, for example, be scrutable only from (hypothetical) evidence which cannot be expressed linguistically.

E could thus be undefinable because it is linguistically, or intentionally, or because it is metaphysically fundamental. It is to be expected that there are expressions of philosophical relevance which fall into one of these categories. But I do not believe that there is a problem for the general project of providing definitions to be found here, because the vast majority of these expressions are not primitive in any of these ways. For a start, it is safe to assume that few philosophical categories are metaphysically fundamental. It is furthermore plausible that in most cases, we are familiar with the kinds of properties on which they supervene, at least in the sense of having intentional access to them. We are thus only left with expressions which are linguistically fundamental despite being intentionally (and metaphysically) non-fundamental.

It is possible that there are such expressions. For example, we might be able to apply some terms only in response to sensory evidence which we are unable to express linguistically. But firstly, even in such cases it is not clear why we should assume that we are unable to express the features in question linguistically even in principle. Secondly, it seems far-fetched to think that many philosophical terms are such that their application is triggered (only) by non-linguistic evidence. Most philosophically relevant properties are not directly perceivable, let alone accessible only via perception. ${ }^{158}$ Finally, the ubiquity of thought experiments in philosophical

${ }^{158}$ Exceptions are arguably provided by expressions related to conscious experience. 
practice indicates that a great number of philosophical terms are not linguistically fundamental. For, in evaluating hypothetical scenarios, we apply the terms in question in response to (hypothetical) evidence which is phrased linguistically.

I just argued that the majority of philosophically relevant expressions are not linguistically fundamental. If an expression is thus non-fundamental, we can describe hypothetical scenarios to which it applies, without using the expression in the descriptions. Theoretically, one could use these descriptions to construct a Carnap sentence for our target expression, call it $\mathrm{T}$, which may roughly look as follows:

$\left.\exists \mathrm{x}\left(\left(\mathrm{D}_{1} \rightarrow \mathrm{F}(\mathrm{x})\right) \&\left(\mathrm{D}_{2} \rightarrow \mathrm{G}(\mathrm{x})\right) \& \ldots\right)\right) \rightarrow \mathrm{T}(\mathrm{x})$

The idea here is to determine for every scenario to which entities the expression in question applies and then to form a disjunction of these applications across the scenarios. However, in order to spell out the content of the target expression in such a way, one would have to invoke infinitely many hypothetical scenarios, resulting in a Carnap sentence of infinite length. Since this obviously exceeds the limits of manageability, an expression's being linguistically non-fundamental does not guarantee that it is definable: We could still be unable to spell out its content in a finite way. Let me give a positive account of how this could be the case: Since T is linguistically non-fundamental, we have a set of other terms (the O-terms) at our disposal by means of which we can express facts (the O-facts) on which the application of $\mathrm{T}$ supervenes a priori. That is to say, each configuration of $\mathrm{O}$-facts determines a priori whether $\mathrm{T}$ applies or not, the application of $\mathrm{T}$ with respect to the various $\mathrm{O}$-facts reflecting $\mathrm{T}$ 's primary intension. Now assume that $\mathrm{T}$ is assigned to the configurations of $\mathrm{O}$-facts completely at random. There is thus no pattern governing the applicability of $\mathrm{T}$ relative to the $\mathrm{O}$-facts. ${ }^{159} \mathrm{In}$ such a case, there is no shorter way of expressing the application conditions of $\mathrm{T}$ by means of O-terms then in the form of a Ramsey sentence of the kind I just outlined. And thus, the existence of a pattern in the relation between the applicability of a term $\mathrm{T}$

${ }^{159}$ Cf. Jackson 2005 for more on such patterns. 
and the facts expressed by other terms is a necessary condition for T's definability.

It is hard to see, however, how this necessary condition could be turned into an objection against the project of providing definitions. It seems hopeless to argue that any terms which we would like to define exhibit such patternlessness. As Jackson notes, we could not possibly have learned to use such a term (cf. Jackson 2005, 128). For in such a case, knowledge of the term's extension with respect to an arbitrarily large number of scenarios would still be insufficient to tell whether it can be applied to any other scenario. Thus, if there was no relevant pattern, we would not be able to apply the term in question to a situation we are not already familiar with. It follows that there has to be a pattern in the application conditions of the terms we use, more specifically a pattern with respect to those properties in response to which we apply them. Let me add to this that since it is usually not overwhelmingly difficult to acquire and apply a term, this pattern will not be overwhelmingly complicated, either.

It is still a theoretical possibility that we are unable to express the pattern in a finite way. But while I see no way to prove that it is possible to do this, a denial of this claim seems excessively pessimistic with respect to the expressive powers of language: If an expression's application supervenes non-randomly on properties which we can express, then why should the pattern in the application conditions not be expressible as well?

In this section, I identified a number of necessary conditions for the definability of an expression. First of all, the definiendum must not be metaphysically, intentionally, or linguistically fundamental. I argued that the majority of philosophically relevant expressions do not fall into either of these categories. Then it turned out that there is another necessary condition on an expression's definability: There must be a pattern in the connection between the definiendum and other terms - which, however, cannot be seriously denied. I conclude that most philosophically relevant expressions meet the identified necessary conditions for their definability, which shows that considerations about the relation between the 
definiendum and everything else provide no principled obstacles to the project of defining philosophical terms.

\subsubsection{Objections from the format of concepts}

It has been argued that the eligibility of definitions depends on the structure or the format of the concept in question. For this reason, (relatively) recent theories of concepts such as the prototype theory are often taken to speak against the prospects of conceptual analysis (cf. for example Kornblith $2007 \mathrm{a}, 41 \mathrm{f}$.). In my view, our concepts come in a variety of formats. One therefore should not expect to find an answer to the question as to which of the theories in question is the correct one. Be that as it may, it still makes sense to discuss possible implications of the structure of a concept on the definability of a term which expresses it.

According to the classical theory of concepts, our concepts have a definitional structure in the sense of being composed of simpler concepts which provide necessary conditions for the application of the concept thus composed. It would obviously be convenient for conceptual analysis if the classical theory was correct. Laurence and Margolis even claim that conceptual analyses require that concepts have definitional structure (cf. Laurence \& Margolis 2005/2011; cf. also Stich 1992; Ramsey 1992). And they go on to argue that since there are good reasons for rejecting the classical theory, the prospects of conceptual analysis are bleak. But this is not a compelling argument; in particular, its first premise seems clearly false: While I agree that only comparably few of our terms have definitional structure in any substantial sense, this is only a problem if one expects analyses to be very short or easy to come by. Definitions in the sense I outlined above only aim at capturing a term's application conditions, not its putative compositional structure.

The prototype theory provides a very different account of concepts (cf. e.g. Rosch \& Mervis 1975). Its basic idea is that the application of a concept is dependent on a putative referent's similarity with a prototype. Let me spell 
this out in a bit more detail: Suppose that a concept is associated with a set of features. That thing which displays these features most distinctively is the prototype of the category in question. Now, the concept applies to anything which has a sufficient number of the associated features, or alternatively to anything whose (weighted) sum of the degrees to which the features apply exceeds a certain value. It is often considered as a major advantage of the theory that it can account for so-called typicality effects: For example, chairs are considered by subjects as more typical pieces of furniture than refrigerators; the former are also more quickly and more accurately categorized as such than the latter. According to prototype theory, this is because chairs are closer to being a prototypical piece of furniture, i.e. they have more of the features which are relevant for membership in the category.

Typicality effects seem to be ubiquitous. Sharon Armstrong et al. have found that they can even be observed with respect to categories like 'even number' (cf. Armstrong, Gleitman \& Gleitman 1983): For example, the number 8 is categorized as an even number more quickly than the number 34 and is also considered as a better example of an even number. However, while such effects may teach us something about the psychological mechanisms involved in categorizations, one should be careful to draw conclusions about the contents of the relevant concepts. The sole criterion for a number's being even is whether it is divisible by 2 . It would be strange to hold that membership in the category depends on, say, similarity to the number $2 .{ }^{160}$

It has been denied that prototypical concepts can be defined because the relevant prototypical features do not yield necessary and sufficient conditions, for example by Stephen Stich (cf. Stich 1992, 249). In one sense, this is correct: For example, it may be that no single feature is necessary for the applicability of the concept. But in another sense, the prototypical features clearly do provide necessary and sufficient conditions. In order to avoid potential terminological confusions about what is meant

${ }^{160}$ Armstrong et al. also take such examples to show that there is no strong connection between a concept's exhibiting typicality effect and its having prototypical structure. 
by necessary and sufficient conditions, let me try to phrase my response to the objection in more neutral terms: Prototype theorists do not deny that concepts have application conditions. I.e., they concede that there are conditions which have to be met by, say, an object or a situation to fall under the concept. Prototype theory even has something to say about the structure of these application conditions, namely that they are determined by a set of features which have to be present to a sufficient degree in a putative referent. ${ }^{161}$

Now, a definition simply aims at capturing these application conditions in an explicit linguistic format. I concede that doing so would often be an extremely tedious task. But I fail to see a principled reason why it should be impossible. One 'only' needs to identify the relevant features and their relative importance for the concept's applicability, which can be done in familiar ways - for example, by considering our judgments about hypothetical cases. ${ }^{162}$ Let me add that it is plausible that when we are dealing with a prototype concept, we will usually be satisfied with an analysis which does less than specifying the concept's application conditions. Often, it will be sufficient to identify the features, or even only the kinds of features, which are relevant for the relevant term's applicability, without determining their relative importance in full detail. Similar things could be said about other theories of concepts: As long as they grant that concepts do have application conditions, which is hard to deny, then there is no apparent reason why these application conditions should not be captured by using strings of words which do not involve the target terms themselves. At best (or worst), some of the formats in which concepts appear provide practical obstacles for specifying their application conditions.

\footnotetext{
${ }^{161}$ Cf. Jackson 1998a, 61 for a similar kind of response.

${ }^{162}$ Since cluster terms like 'water' can at least arguably be understood as prototypical, my discussion in chapter 3 bears some relevance to the question how this can be done.
} 


\subsubsection{The absence of successful definitions and some reasons for optimism}

I just discussed a number of objections to the eligibility of definitions. It emerged that there are no principled reasons for thinking that definitions cannot be had. However, we found that there are a number of practical obstacles to the task of providing them. In the following, I will determine a couple of other factors which complicate the search for definitions and which may therefore help to explain why adequate definitions are so hard to come by. Against this backdrop, I will discuss to what extent the striking absence of successful definitions gives us reason to be skeptical with respect to the prospects of finding definitions and I will try to provide some reasons for optimism.

One merely practical reason why successful definitions are so rare is that philosophers usually aim at short definitions and rarely offer analyses which exceed a certain length. It is of course reasonable to prefer short definitions if they can be found - simplicity should be a virtue for definitions, too. But firstly, as I argued above one should not require them to be short. And secondly, there is no particular reason to expect that very short definitions are to be had $-{ }^{163}$ this assumption seems to derive its popularity mainly from an adherence to the classical theory of concepts.

Another factor which might hinder the project of constructing definitions is that speakers may associate slightly different properties with an expression. In fact, for many concepts it would be rather surprising if it turned out that all competent speakers associate exactly the same primary intension with them (cf. also Jackson 2005, 135). Such variation in primary intensions can result in disagreement in the evaluation of one or more relevant hypothetical scenarios which can in turn lead to disagreement about the adequacy of a proposed definition. It can thus happen that a definition perfectly reflects the term's application conditions in a subject's idiolect and yet fails to be accepted by others. So what should be done in such a case? If it can be established that the disagreement about the adequacy of

${ }^{163}$ Jackson elaborates a bit more on this point in Jackson 2005, 132. 
the definition is just verbal in this sense, one can either accept the definition, and maybe go on constructing definitions which reflect other people's concepts as well, or - which seems more reasonable - try to identify a core which is common to the concepts of all or at least most competent speakers. This latter proposal would inter alia be in line with Jackson's idea that conceptual analysis aims at analyses of our folk concepts. But either way, the fact that there can be mild intersubjective variation in the properties associated with a term does not amount to a principled obstacle to the task of providing definitions, either.

The main reason why many philosophers are skeptical with regard to the chances of providing definitions is that there are hardly any examples of adequate definitions which have been yielded so far. However, the preceding considerations have not only shown that there are no principled reasons to think that explicit analyses cannot be given, but also that there are a number of purely practical hindrances to that task. This result alleviates the objection from the absence of definitions. But needless to say, the only thing which will convince the skeptic is at least one clear-cut example of a waterproof analysis of a philosophically relevant term. Unfortunately, I cannot offer such an analysis here (although I will bring in an analysis which I think holds some promise). In the remaining part of this chapter I will, however, try to provide some support to the idea that the project of searching for definitions is a progressing one, rather than a degenerate one.

The objection from the absence of definitions should plausibly be construed as a kind of pessimistic induction: Since all the previous attempts to define key philosophical terms have failed, it is very likely that future attempts will fail as well. This thought is present in the comment of Williamson I quoted near the beginning of this chapter and also in the following remark of Chalmers and Jackson on the analysis of knowledge:

On the absence of explicit analyses of knowledge: We take it that this is a reasonable conclusion to draw from four decades of failed attempts to produce explicit analyses. Certainly no explicit analysis has met with widespread 
approval, and proposed analyses are always confronted quickly by plausible counterexamples. (Chalmers \& Jackson 2001, 321)

I am not sure about their claim that proposed definitions are always confronted by plausible counterexamples. But admittedly, counterexamples to such proposals are often found quickly. On the other hand, this fact also gives some reasons for optimism. For, it suggests that we are rather good at identifying weak spots in a proposed analysis, which is plausibly an important condition for making progress. And in many cases, such counterexamples do not just reveal yet another failure. Often, there is a lot to be learned from them. The failure of the standard analysis of knowledge presents a particularly vivid example for this: For it was quickly found that in all the counterexamples to the standard analysis, the subject's belief falls short of being knowledge because it owes its truth to luck in some way or other. And while a definition of the sort ' $\mathrm{S}$ knows that $\mathrm{p}==_{\mathrm{df}} \mathrm{S}$ has the justified true belief that $p$ the truth of which was not arrived at by luck' is surely unsatisfactory because it still needs to be specified what 'luck' exactly means, the insight does reveal that there is an important pattern which underlies the counterexamples. In my view, a lot of progress has been made in the attempt to capture this pattern in recent years.

One approach to explicating this pattern involves the notion of safety: A belief, for example, is safe if it could not have easily been false, that is if it is not only in fact true but also in all close worlds. Now a comparably recent idea to take this notion as the basis of an analysis of knowledge goes roughly as follows:

(MS) $\mathrm{S}$ knows that $\mathrm{p}={ }_{\mathrm{df}} \mathrm{S}$ has the true belief that $\mathrm{p}$ which was produced by a safe process or method. ${ }^{164}$

Even though this analysis does get a significant number of problem cases right, it is, needless to say, far from being generally accepted. It is also true that a number of putative counterexamples to it have been proposed (cf. Comesaña 2005; Kemp 2009). But even so, there is still plenty of room for

${ }^{164}$ Here, (MS) stands for Method Safety. A proposal which can be construed along these lines was made by Mark Sainsbury (cf. Sainsbury 1997). 
improving a safety-based account. Comesaña's and Kemp's cases are such that a method which (arguably) produces a knowledgeable belief is not safe because it could have easily been used in circumstances in which it would have been unreliable. At the same time, that method is highly reliable in the circumstances present during its actual application. One might thus propose to modify the analysis at issue slightly, yielding:

(MS*) $\mathrm{S}$ knows that $\mathrm{p}={ }_{\mathrm{df}} \mathrm{S}$ has the true belief that $\mathrm{p}$ which was produced by a process or method which is generally reliable and safe in the circumstances in which it produced the belief. ${ }^{165}$

This formulation is still not perfect, because it seems to misclassify the subject's true belief that she sees a barn in Fake Barn County (cf. Goldman 1976, 772f.) as knowledge: For it would seem that it is a part of the circumstances in which the subject forms her belief that the light conditions are good, she is not distracted, and that there is a real barn standing in front of her. Given this, the belief conforms to (MS*). ${ }^{166}$ Plausibly, the subject's belief in the fake barn scenario is not considered as knowledge because the recognition of barns via visual perception, while being generally reliable, is unreliable in the subject's current environment. So the above proposal can be repaired by adding a clause concerning the local reliability of the process or method involved, which is anyway an independently plausible condition for knowledge: ${ }^{167}$

(MS**) S knows that $\mathrm{p}={ }_{\mathrm{df}} \mathrm{S}$ has the true belief that $\mathrm{p}$ which was produced by a process or method which is generally and locally reliable and safe in the circumstances in which it produced the belief.

This proposal handles a number of the most persistent problem cases for an analysis of knowledge, such as the original Gettier scenarios, various lottery cases, and - as we just saw - the fake barn scenario. Despite this, I

\footnotetext{
165 Maybe surprisingly, the resulting view closely resembles Sosa's virtueepistemologist account which he develops in Sosa 2007.

${ }^{166}$ Sosa is happy to accept this consequence of his account (cf. the previous footnote) and holds that the subject in the scenario does know that there is a barn in front of her. ${ }^{167}$ Here, local reliability should be understood not as a spatial, but as a modal notion.
} 
am not claiming that I have thus presented the ultimate analysis of knowledge. It will probably turn out that this proposal still needs to be refined or that other accounts which do not rely on the notion of safety are more suitable. I mainly wanted to illustrate that there are approaches which integrate many lessons from counterexamples to previous analyses, which have already yielded analyses which are (at least in an intuitive sense) more adequate than the standard analysis of knowledge and which are eligible for further development. I therefore think there is hope that the program of constructing definitions is progressing.

This chapter was dedicated to a wide range of goals which people pursue when they practice conceptual analysis and to an assessment of the preconditions and the prospects for achieving these goals.

In the first part of the chapter, I discussed various ways in which the method and the aims of conceptual analysis have been understood: As a tool for revealing the essences of philosophically relevant categories, as an essential ingredient in reductive explanations, as a way of locating higherlevel phenomena in a fundamental picture of the world, etc. It transpired that conceptual analysis can be a useful tool even when it fails to deliver explicit analyses, or does not even aim at delivering them. But a number of the ways of understanding conceptual analysis which I discussed do, or at least may, involve definitions at some stage or other.

The second part of the chapter was therefore meant to provide an assessment of the prospects of the project of constructing definitions of philosophically relevant terms. After determining adequacy conditions for definitions, I discussed potential obstacles to their achievability. Although there are a number of considerations which suggest that it will be difficult to develop definitions which are not susceptible to counterexamples, I found no principled reason for thinking that it is impossible. It is undeniable that the project of providing analyses has few spectacular successes to present as yet. Nevertheless, there is evidence that it is progressing. 
